\title{
CP-violation hypothesis and the relativistic electromagnetic structure of nucleons
}

\author{
A.F. Krutov \\ Samara State University, Samara, Russia \\ E-mail: krutovassu.samara.ru \\ M.Yu. Kudinov* \\ Samara State University, Samara, Russia \\ E-mail: kudinovessu.samara.ru
}

In this paper we propose to discuss the electromagnetic structure of the nucleon in the framework of the violation hypothesis of discrete symmetries. Analysis is performed with help of the general method of the Lorentz-covariant local operator matrix element parametrization. The proposed analysis allows to solve the problem of the "non-Rosenbluth" behavior of the electromagnetic proton form factors. It is shown that the $C P$-violation effects give rise to the additional anapole proton form factor.

The XXth International Workshop High Energy Physics and Quantum Field Theory September 24 - October 1, 2011

Sochi, Russia

${ }^{*}$ Speaker. 


\section{Introduction}

One of the most interesting experimental results of the recent time is the "non-Rosenbluth" behavior of the proton electromagnetic form factors in the scattering of the longitudinal polarized electron beams by the protons $[1,2,3,4]$. Ratio of the electric form factor to magnetic one was obtained in these experiments as linearly decreased function of the momentum transfers square. This result is in conflict with results of the nonpolarized electrons scattering by proton (see Fig.1). In these experiments the electromagnetic proton form factors are measured by the Rosenbluth's method (see, e.g.[5, 6]), which gives for this ratio unity approximately [7]. Let us remark that the replicates of the proton form factors measurements by the Rosenbluth's method were performed recently [8] and these measurements have supported the previous results [7]. So the manifest conflict between the experiments on the elastic ep-scattering with polarized and nonpolarized electrons is well-established.

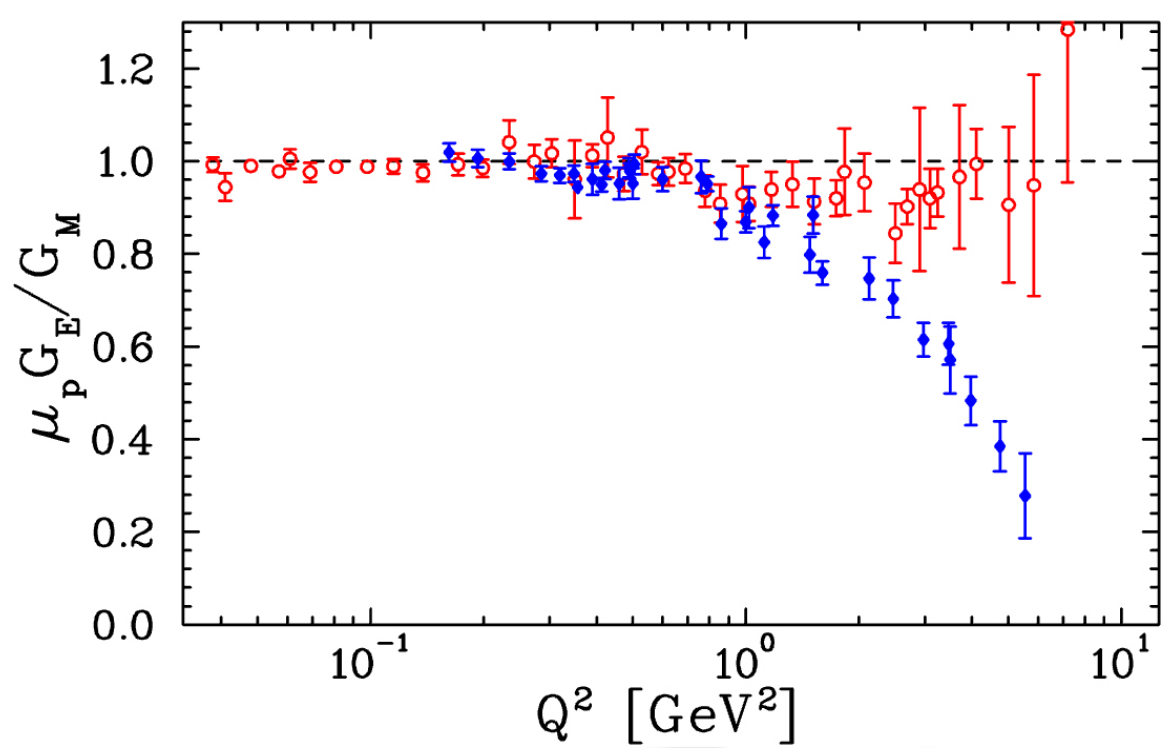

Figure 1: "Non-Rosenbluth" behavior of the proton electromagnetic form factors (the blue points) and Rosenbluth behavior (the red points). The data are taken from [11].

One of the basic theoretical approach to the elimination of this contradiction is taking into account two-photon exchange in the electro-proton scattering [9]. However this approach, at first, does not eliminate this conflict completely (see Fig.2-a)) $[9,10]$ and, secondly, causes the new contradiction. This new contradiction implies that the two-photon exchange predicts the essential difference between results of the electron-proton and positron-proton scattering [11], but direct experimental comparison between these processes does not established any charge-asymmetry of this kind (see Fig.2-b)) [10].

So the "non-Rosenbluth" behavior problem can't be considered as the full solved.

In this work we suggest to analyse the results of the elastic ep-scattering in the framework of the hypothesis about $C P$-violation in the proton as composite system with strong interaction. The hypothesis about $C P$-violation in the electromagnetic processes in these systems was proposed 


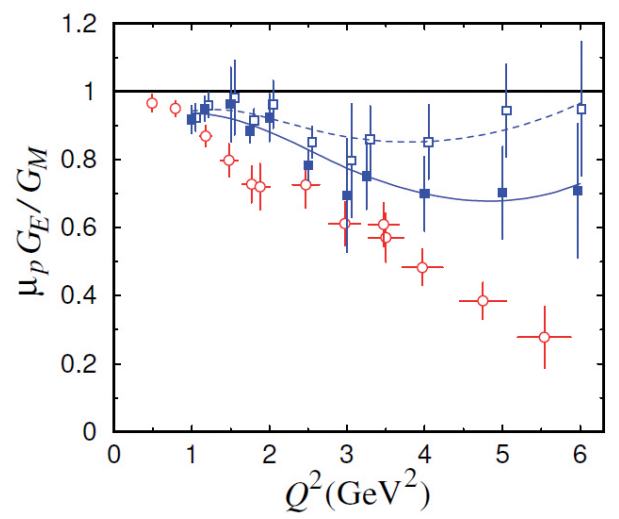

a)

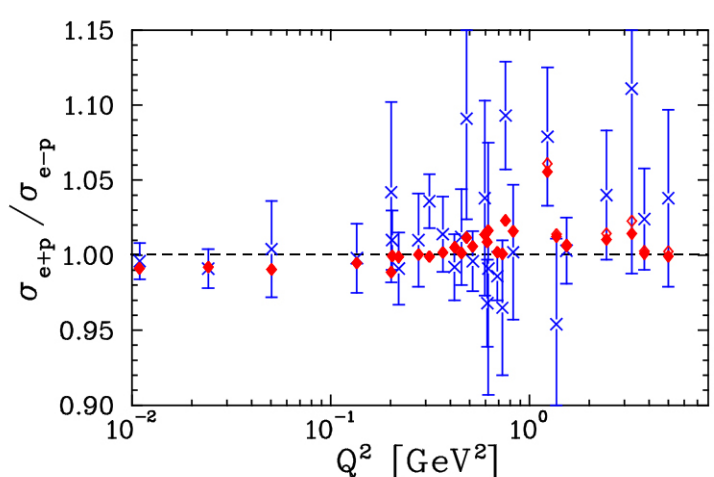

b)

Figure 2: a) Conventional approach to the solution of this problem is two-photon exchange. b) Asymmetry between $e^{-} p$ - and $e^{+} p$-scattering.

for a long time (see, e.g. [12, 13, 14]). Our paper is close on spirit to paper [14], in which the possible $C P$-violation in the elastic $e p$-scattering is related with the availability of the so called anapole proton form factor in the matrix element of the electromagnetic proton current. In the present paper we use the mathematical apparatus from [13], where the possible $C P$-violation in elastic electron scattering on deuteron as composite nucleon system was considered.

\section{Calculations}

For analysis we used special construction method of the proton electromagnetic current operator (see, e.g. [17]) which follows from the general method of relativistic invariant parameterization for local operator matrix elements proposed by Cheshkov and Shirokov [18]. In fact, this parametrization is a realization of the Wigner-Eckart theorem for the Poincare group and so it enables one for given matrix element of arbitrary tensor dimension to separate the reduced matrix elements (form factors) which are invariant under the Poincaré group. The matrix element of a given operator is represented as a sum of terms, each one of them is a covariant part multiplied by an invariant part. In such representation a covariant part describes transformation (geometrical) properties of the matrix element, while all the dynamical information on the transition is contained in the invariant part - reduced matrix elements.

The matrix element of electromagnetic current with regard to the self-adjointness, the current conservation low and $C P$-symmetry:

$$
\begin{aligned}
& \left\langle\vec{p}, m\left|j_{\mu}(0)\right| \vec{p}^{\prime}, m^{\prime}\right\rangle=\sum_{m^{\prime \prime}}\left\langle m\left|D^{1 / 2}\left(p, p^{\prime}\right)\right| m^{\prime \prime}\right\rangle \times \\
& \quad \times\left\langle m^{\prime \prime}\left|f_{10}\left(Q^{2}\right) K_{\mu}^{\prime}+i f_{30}\left(Q^{2}\right) R_{\mu}\right| m^{\prime}\right\rangle
\end{aligned}
$$

where $\vec{p}^{\prime}, \vec{p}$ are 3-momenta of particle in the initial and the final states, respectively. $m^{\prime}, m$ are spin projections, 4 -vectors in the right side are determined by follows:

$$
K_{\mu}^{\prime}=\left(p+p^{\prime}\right)_{\mu}, R_{\mu}=\varepsilon_{\mu \nu \lambda \rho} p^{v} p^{\prime \lambda} \Gamma^{\rho}\left(p^{\prime}\right),
$$


here $\varepsilon_{\mu \nu \lambda \rho}$ is a completely anti-symmetric pseudo-tensor in four dimensional space-time with $\varepsilon_{0123}=-1, \Gamma^{\mu}(p)$ is relativistic spin operator (see, e.g. [19]), $f_{10}\left(Q^{2}\right), f_{30}\left(Q^{2}\right)$ are electric and magnetic form factors, respectively.

The form factors from Eq. (2.1) are connected with Sach's ones [20]:

$$
f_{10}\left(Q^{2}\right)=\frac{2 M G_{E}\left(Q^{2}\right)}{\sqrt{4 M^{2}+Q^{2}}}, \quad f_{30}\left(Q^{2}\right)=-\frac{4 G_{M}\left(Q^{2}\right)}{M \sqrt{4 M^{2}+Q^{2}}} .
$$

Using Eqs. (2.1) and (2.3) it is possible to obtain the conventional Rosenbluth's cross section for elastic nonpolarized $e p$-scattering (see, e.g., [5]):

$$
\frac{d \sigma}{d \Omega}=\left(\frac{d \sigma}{d \Omega}\right)_{M o t t}\left[A\left(Q^{2}\right)+B\left(Q^{2}\right) \tan ^{2}\left(\frac{\theta}{2}\right)\right]
$$

where $\theta$ is the scattering angle of electrons in the laboratory frame, $E$ is energy of electron in the initial state, $t=\left(p-p^{\prime}\right)^{2}=-Q^{2}$ is the momentum transfer square.

And ratio of electric and magnetic form factor in the polarized ep-scattering take the form:

$$
\frac{R\left(Q^{2}\right)}{\mu_{p}}=\frac{G_{E}\left(Q^{2}\right)}{G_{M}\left(Q^{2}\right)}=-\frac{P_{t}}{P_{l}} \frac{\left(E+E^{\prime}\right)}{2 M} \tan \left(\frac{\theta}{2}\right),
$$

where $\mu_{p}$ is the proton magnetic moment, $P_{t}, P_{l}$ are transversal and longitudinal polarizabilities of the recoil proton, respectively. $E^{\prime}$ is the energy of electrons in the final state.

The cross section of electron scattering on the spiless and structureless particle (Moth's section) and the invariant function of the momentum transfer square have the conventional expressions:

$$
\begin{gathered}
\left(\frac{d \sigma}{d \Omega}\right)_{M o t t}=\frac{\alpha^{2} \cos ^{2}(\theta / 2)}{4 E^{2} \sin ^{4}(\theta / 2)\left[1+2 \xi \sin ^{2}(\theta / 2)\right]}, \\
A\left(Q^{2}\right)=\frac{G_{E}^{2}\left(Q^{2}\right)+\tau G_{M}^{2}\left(Q^{2}\right)}{1+\tau}, \\
B\left(Q^{2}\right)=2 \tau G_{M}^{2}\left(Q^{2}\right),
\end{gathered}
$$

where $\xi=E / M, \tau=-Q^{2} / 4 M^{2}=t / 4 M^{2}$.

In the framework of the $C P$-violation hypothesis the parametrization of the electromagnetic current matrix element for proton with regard to the self-adjointness, the current conservation low and $C P$-violation will take form:

$$
\begin{aligned}
& \left\langle\vec{p}, m\left|j_{\mu}(0)\right| \vec{p}^{\prime}, m^{\prime}\right\rangle=\sum_{m^{\prime \prime}}\left\langle m\left|D^{1 / 2}\left(p, p^{\prime}\right)\right| m^{\prime \prime}\right\rangle\left\langle m^{\prime \prime}\right|\left[f_{10}\left(Q^{2}\right) K_{\mu}^{\prime}+\right. \\
& \left.+f_{11}\left(Q^{2}\right)\left(i p_{\mu} \Gamma^{\mu}\left(p^{\prime}\right)\right) K_{\mu}^{\prime}+f_{20}\left(Q^{2}\right) A_{\mu}+i f_{30}\left(Q^{2}\right) R_{\mu}\right]\left|m^{\prime}\right\rangle
\end{aligned}
$$

where

$$
A_{\mu}=\Gamma_{\mu}\left(p^{\prime}\right)-\left(\frac{K_{\mu}^{\prime}}{K^{\prime 2}}+\frac{K_{\mu}}{K^{2}}\right)\left(p_{\lambda} \Gamma^{\lambda}\left(p^{\prime}\right)\right), \quad K_{\mu}=\left(p-p^{\prime}\right)_{\mu}
$$

$f_{11}\left(Q^{2}\right)$ - electric dipole form factor, $f_{20}\left(Q^{2}\right)$ - anapole form factor 
By analogy with obtaining Eq. (2.4) it is possible to calculate the cross section with help of Eq. (2.9) taking into account $C P$-violation:

$$
\begin{aligned}
& \frac{d \sigma}{d \Omega}=\left(\frac{d \sigma}{d \Omega}\right)_{M o t t}\left[a\left(Q^{2}\right)+b\left(Q^{2}\right) \tan ^{2}\left(\frac{\theta}{2}\right)+\right. \\
& \left.+f_{11}\left(Q^{2}\right) f_{20}\left(Q^{2}\right) D(\tau, \theta)+f_{20}^{2}\left(Q^{2}\right) F(\tau, \theta)\right],
\end{aligned}
$$

where

$$
\begin{gathered}
a\left(Q^{2}\right)=\frac{g_{E}^{2}\left(Q^{2}\right)+\tau g_{M}^{2}\left(Q^{2}\right)}{1+\tau}+f_{11}^{2}\left(Q^{2}\right) \tau M^{2}(1+\tau), \\
b\left(Q^{2}\right)=2 \tau g_{M}^{2}\left(Q^{2}\right), \\
F(\tau, \theta)=\frac{x}{2 \sqrt{\tau}(\tau+1)}\left(\sqrt{\frac{1}{x}+\tau+1}+2 \sqrt{\tau}(\tau+1)\right), \\
D(\tau, \theta)=\frac{M^{5}\left(E+E^{\prime}\right)\left(\xi-\xi^{\prime}+8 \tau+10 \tau \xi\right)}{8}(1+x(1+2 \xi)), \\
x=\tan ^{2}(\theta / 2), \quad \xi^{\prime}=\frac{E^{\prime}}{M},
\end{gathered}
$$

$g_{E}^{2}\left(Q^{2}\right), g_{M}^{2}\left(Q^{2}\right)$ are electric and magnetic proton form factor, respectively, with regard to $C P$ violation, $x=\tan ^{2}(\theta / 2)$. All other notations coincide with ones in Eqs. (2.4) - (2.8).

Analogy of the expression (2.5) with regard to $C P$-violation has following form:

$$
\begin{gathered}
\frac{P_{l}}{P_{t}}=-\frac{g_{M}\left(Q^{2}\right)}{g_{E}\left(Q^{2}\right)} \frac{\left(E+E^{\prime}\right)}{2 M} \tan \left(\frac{\theta}{2}\right) \times \\
\times\left[\frac{1+\alpha f_{20}^{2}\left(Q^{2}\right) / g_{M}^{2}\left(Q^{2}\right)}{1+\beta\left(f_{11}\left(Q^{2}\right) f_{20}\left(Q^{2}\right)\right) /\left(g_{M}\left(Q^{2}\right) g_{E}\left(Q^{2}\right)\right)}\right],
\end{gathered}
$$

where

$$
\alpha=\frac{\sqrt{\tau+1}}{8 \sqrt{x} \tau(\sqrt{x \tau}+\sqrt{x \tau+x+1})}, \quad \beta=\frac{1}{M^{2}(\tau+1)} .
$$

\section{Approximations}

In this Section we'll discuss the approximations in which the analysis of the elastic epscattering will be produced with help of Eqs.(2.11) - (2.16).

\subsection{EDM}

The measurements of the neutron dipole moment in the modern experiments give the very small upper limit of this value $[15,16]$.

In this connection it is naturally to suppose that the electric dipole form factor in the Eqs. (2.11) - (2.16) can be assumed equal zero approximately:

$$
f_{11}\left(Q^{2}\right) \approx 0 \text {. }
$$


Cross section of the non-polarized $e p$-scattering in this approximation:

$$
\frac{d \sigma}{d \Omega}=\left(\frac{d \sigma}{d \Omega}\right)_{M o t t}\left[a\left(Q^{2}\right)+b\left(Q^{2}\right) \tan ^{2}\left(\frac{\theta}{2}\right)+g_{A}^{2}\left(Q^{2}\right) F(\tau, \theta)\right],
$$

where functions $a\left(Q^{2}\right)$ and $b\left(Q^{2}\right)$ have the structure of Eqs. (2.7) and (2.8), respectively, with substitution of $G_{E, M}\left(Q^{2}\right)$ by $g_{E, M}\left(Q^{2}\right)$.

$$
f_{20}\left(Q^{2}\right)=\frac{g_{A}\left(Q^{2}\right)}{\sqrt{1+\tau}} .
$$

Equation (2.16) in this approximation has following form:

$$
\begin{aligned}
\frac{P_{l}}{P_{t}} & =-\frac{g_{M}\left(Q^{2}\right)}{g_{E}\left(Q^{2}\right)} \frac{\left(E+E^{\prime}\right)}{2 M} \tan \left(\frac{\theta}{2}\right) \times \\
& \times\left[1+\frac{\alpha}{1+\tau} \frac{g_{A}^{2}\left(Q^{2}\right)}{g_{M}^{2}\left(Q^{2}\right)}\right],
\end{aligned}
$$

\subsection{Kinematical function}

Let us discuss now the kinematical function $F(\tau, \theta)$ :

$$
F(\tau, \theta)=\frac{x}{2 \sqrt{\tau}(\tau+1)}\left(\sqrt{\frac{1}{x}+\tau+1}+2 \sqrt{\tau}(\tau+1)\right),
$$

This function has the linear asymptotic $F_{a}(\tau, x)$ at large $x=\tan ^{2}(\theta / 2)$ :

$$
F_{a}(\tau, x)=x\left(1+\frac{1}{2 \sqrt{\tau(\tau+1)}}\right)+\frac{1}{4 \sqrt{\tau}(\tau+1)^{3 / 2}} .
$$

We can easily to see (Fig.3) that the function $F(\tau, \theta)$ can be substituted by the asymptotic $F_{a}(\tau, x)$ to a quite good approximation in the region of the not very small $\tau$ and for the angles $(x \geq 0.5)$. These angles are typical for the elastic ep-scattering. It should be mentioned that the quality of this approximation is improved with increasing $\tau$, e.g. with increasing momentum transfer square. To demonstrate the quality approximation let us construct the function:

$$
S(\tau, x)=\left|\frac{F(\tau, \theta)-F_{a}(\tau, x)}{F(\tau, x)}\right| .
$$

This function take the following values at different parameters. At $\tau=0.01,\left(Q^{2}=0.035\right.$ $\left.\mathrm{GeV}^{2}\right), x=0.5\left(\theta \approx 70.5^{0}\right) S(\tau, x)=0.14$, and at $x=0.8\left(\theta \approx 83.6^{0}\right) S(\tau, x)=0.072$; at $\tau=0.1$ $\left(Q^{2}=0.352 \mathrm{GeV}^{2}\right), x=0.5 S(\tau, x)=0.098$, and $x=0.8 S(\tau, x)=0.05$. Functions $F(\tau, x)$ and $F_{a}(\tau, x)$ are shown on Fig. 3. for different $\tau$.

So in the region of the transfer momenta in the experiments $[1,2,3,4]$ the function $F(\tau, x)$ from (2.14) can be approximated by function $F_{a}(\tau, x)$ from (3.6) with good accuracy. It should be pointed out that in this approximation the cross section in Eq. (3.2) have the Rosenbluth's structure (2.4) but with other invariant functions:

$$
\frac{d \sigma}{d \Omega}=\left(\frac{d \sigma}{d \Omega}\right)_{M o t t}\left[a\left(Q^{2}\right)+g_{A}^{2}\left(Q^{2}\right) c(\tau)+\left(b\left(Q^{2}\right)+g_{A}^{2}\left(Q^{2}\right) d\left(Q^{2}\right)\right) \tan ^{2}\left(\frac{\theta}{2}\right)\right],
$$




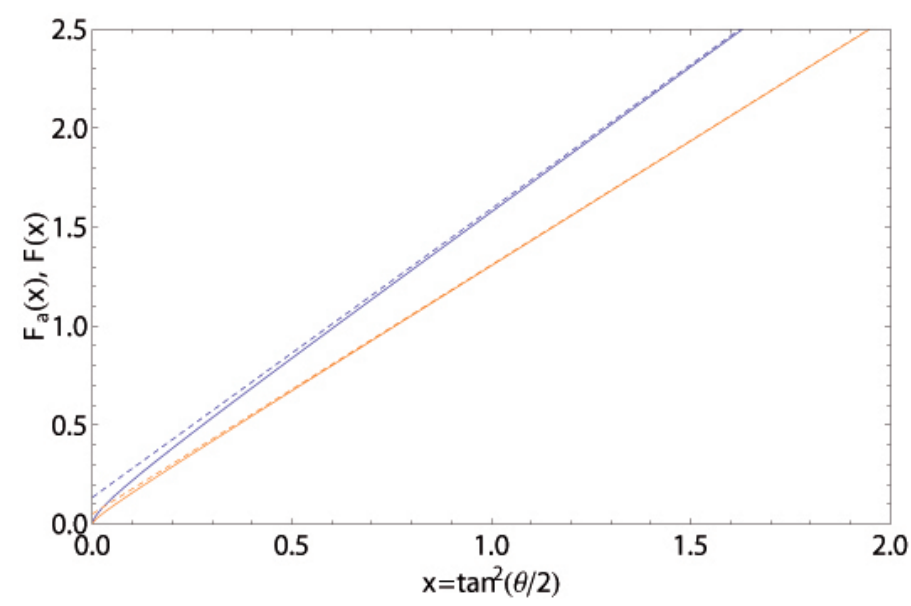

Figure 3: In the region of the experimental angles kinematical function (3.5) is close to linear function $F_{a}$ (3.6).

where

$$
\begin{gathered}
a\left(Q^{2}\right)=\frac{g_{E}^{2}\left(Q^{2}\right)+\tau g_{M}^{2}\left(Q^{2}\right)}{1+\tau}, \quad b\left(Q^{2}\right)=2 \tau g_{M}^{2}\left(Q^{2}\right), \\
c\left(Q^{2}\right)=\frac{1}{4 \sqrt{\tau}(\tau+1)^{5 / 2}}, \quad d\left(Q^{2}\right)=\frac{1}{\tau+1}+\frac{1}{2 \sqrt{\tau}(\tau+1)^{3 / 2}},
\end{gathered}
$$

So effects of the $C P$-violation does not change the Rosenbluth's structure of the cross section which is observed in the non-polarized experiments.

\subsection{Polarized scattering}

We are coming now to the approximations in Eq. (3.4) for the polarized $e p$-scattering. The ratio of the anapole form factor to the magnetic one can being considered as equal to zero approximately at small momentum transfers because in this region conflict between the polarized and non-polarized experiments is absent and contribution of the anapole form factor is present in minor. In the region of the large momentum transfers function $\alpha(\tau, x)$ is very small. In these approximations taking into account (3.3) the following inequality is true for the all region of the momentum transfers:

$$
\frac{\alpha}{1+\tau} \frac{g_{A}^{2}\left(Q^{2}\right)}{g_{M}^{2}\left(Q^{2}\right)} \ll 1
$$

Taking into account the Eqs. (3.1) and (3.11) the Eq. (2.16) coincides fully with Eq. (2.5) obtained in the case of $C P$-conservation with substitution $G_{E, M}\left(Q^{2}\right)$ by $g_{E, M}\left(Q^{2}\right)$ :

$$
\frac{P_{l}}{P_{t}}=-\frac{g_{M}\left(Q^{2}\right)}{g_{E}\left(Q^{2}\right)} \frac{\left(E+E^{\prime}\right)}{2 M} \tan \left(\frac{\theta}{2}\right) .
$$

\section{Cross section and new form factor}

\subsection{Cross section}

After using the above approximations cross section in the hypothesis of $C P$-violation are as follows: 


$$
\begin{aligned}
& \frac{d \sigma}{d \Omega}=\left(\frac{d \sigma}{d \Omega}\right)_{M o t t}\left[a\left(Q^{2}\right)+g_{A}^{2}\left(Q^{2}\right) c(\tau)+\right. \\
& \left.+\left(b\left(Q^{2}\right)+g_{A}^{2}\left(Q^{2}\right) d\left(Q^{2}\right)\right) \tan ^{2}\left(\frac{\theta}{2}\right)\right]
\end{aligned}
$$

where

$$
\begin{gathered}
a\left(Q^{2}\right)=\frac{g_{E}^{2}\left(Q^{2}\right)+\tau g_{M}^{2}\left(Q^{2}\right)}{1+\tau}, \quad b\left(Q^{2}\right)=2 \tau g_{M}^{2}\left(Q^{2}\right), \\
c\left(Q^{2}\right)=\frac{1}{4 \sqrt{\tau}(\tau+1)^{5 / 2}}, \quad d\left(Q^{2}\right)=\frac{1}{\tau+1}+\frac{1}{2 \sqrt{\tau}(\tau+1)^{3 / 2}}, \\
\frac{P_{l}}{P_{t}}=-\frac{g_{M}\left(Q^{2}\right)}{g_{E}\left(Q^{2}\right)} \frac{\left(E+E^{\prime}\right)}{2 M} \tan \left(\frac{\theta}{2}\right) .
\end{gathered}
$$

\subsection{The new values of form factors}

Connection between the new form factors and the measured in experiments functions $R\left(Q^{2}\right), A\left(Q^{2}\right)$ and $B\left(Q^{2}\right)$ :

$$
\begin{gathered}
R\left(Q^{2}\right)=\mu_{p} \frac{g_{E}\left(Q^{2}\right)}{g_{M}\left(Q^{2}\right)}=1-0.13\left(Q^{2}-0.04\right) . \\
(1+\tau) A\left(Q^{2}\right)=g_{E}^{2}\left(Q^{2}\right)+\tau g_{M}^{2}\left(Q^{2}\right)+g_{A}^{2}\left(Q^{2}\right)(1+\tau) c\left(Q^{2}\right), \\
B\left(Q^{2}\right)=2 \tau g_{M}^{2}\left(Q^{2}\right)+g_{A}^{2}\left(Q^{2}\right) d\left(Q^{2}\right) .
\end{gathered}
$$

The new values of proton form factors from expression (4.5) can be rewritten in terms of the Sach's form factors obtained from experiment by Rosenbluth's method (2.7), (2.8):

$$
\begin{gathered}
g_{A}^{2}\left(Q^{2}\right)=\frac{2 \tau(\tau+1)\left(G_{E}^{2}\left(Q^{2}\right)-G_{M}^{2}\left(Q^{2}\right)\left(R\left(Q^{2}\right) / \mu_{p}\right)^{2}\right)}{d\left(Q^{2}\right)-2 \tau(\tau+1) c\left(Q^{2}\right)}, \\
g_{M}^{2}\left(Q^{2}\right)=G_{M}^{2}\left(Q^{2}\right)+g_{A}^{2}\left(Q^{2}\right) \frac{d\left(Q^{2}\right)}{1+\tau}, \\
g_{E}^{2}\left(Q^{2}\right)=g_{M}^{2}\left(Q^{2}\right)\left(\frac{R\left(Q^{2}\right)}{\mu_{p}}\right)^{2} .
\end{gathered}
$$

For the numerical estimation of the proton electromagnetic form factors with consideration of the $C P$-violation we will use the analytical formulas for $G_{E}^{2}\left(Q^{2}\right)$ and $G_{M}^{2}\left(Q^{2}\right)[21,22]$ which fit the experimental data for $A\left(Q^{2}\right)$ and $B\left(Q^{2}\right)$, obtained from Rosenbluth-type experiments.

The results of the our calculation with analytical formulas for $G_{E}^{2}\left(Q^{2}\right)$ and $G_{M}^{2}\left(Q^{2}\right)$ from [22] are presented in Figs. $4-6$.

As it seen from figures the possible effects of the $C P$-violation leads to the small variation of the magnetic form factor and modify the electric form factor widely enough. The anapole form factor on the Fig. 4 is presented in the region where the fit for ratio of the polarizabilities (4.5) is worked. The values of the all form factors depend on the variation of this fit. From these figures we notice that the value of the anapole form factor is much less then the magnetic form factor. This fact justifies our approximation in Eq. (3.11). The anapole form factor has the zero value at the zero momentum transfers, what means that anapole proton moment equals to zero. In this work the 


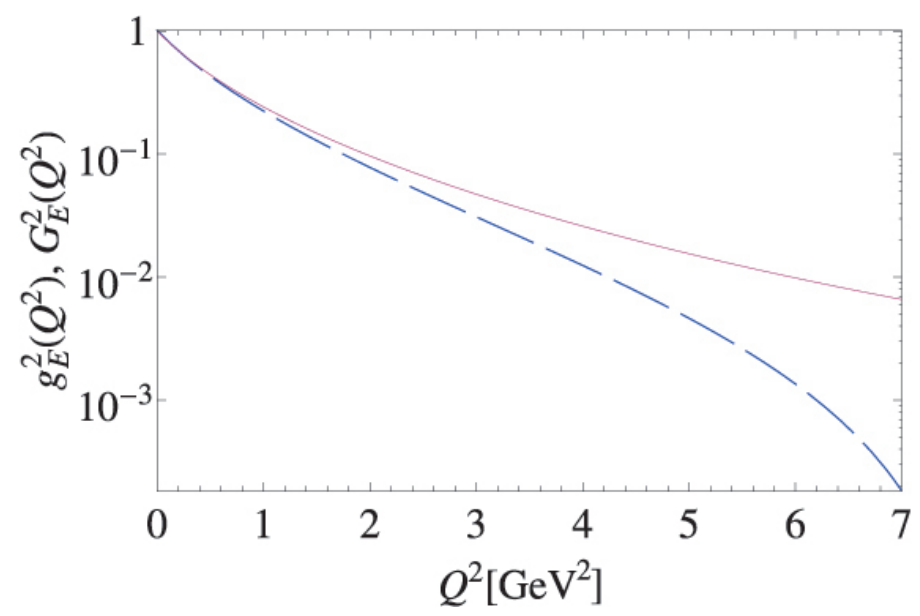

Figure 4: The electric proton form factor. The solid (red) line - $G_{E}^{2}\left(Q^{2}\right)$ from Eq. (2.7) calculated without $C P$-violation, dashed (blue) line - form factor $g_{E}^{2}\left(Q^{2}\right)$ obtained having regard to $C P$-violation effects. For $G_{E}^{2}\left(Q^{2}\right)$ the fit from [22] is used.

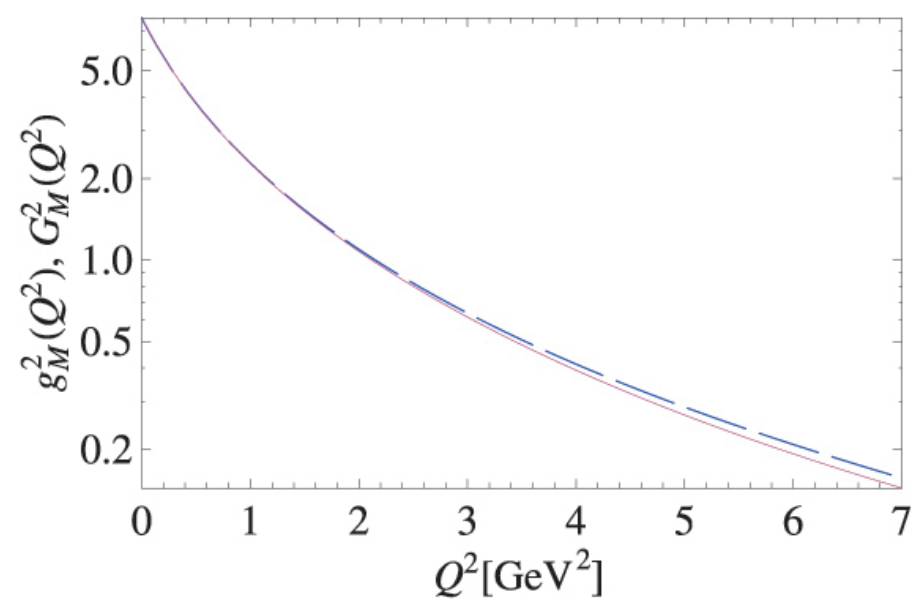

Figure 5: The magnetic proton form factor. The solid (red) line - $G_{M}^{2}\left(Q^{2}\right)$ from Eq. $(2.7,2.8)$ calculated without $C P$-violation, dashed (blue) line - form factor $g_{M}^{2}\left(Q^{2}\right)$ obtained having regard to $C P$-violation effects. For $G_{M}^{2}\left(Q^{2}\right)$ the fit from [22] is used.

proton is considered as composite quark system. Of interest is the description of the $C P$-violation effects in the electromagnetic processes with the participation of the proton in framework of the composite quark model. It will be done in the other work.

\section{Conclusions}

In the paper the analysis of the experimental data in the elastic electron-proton scattering is proposed in the framework of the hypothesis about $C P$-violation in the electromagnetic processes in the composite systems with strong interaction. Analysis is performed with help of the general method of the Lorentz-covariant parametrization of local operator matrix element. The proposed analysis allows to solve the problem of the "non-Rosenbluth" behavior of the electromagnetic pro- 


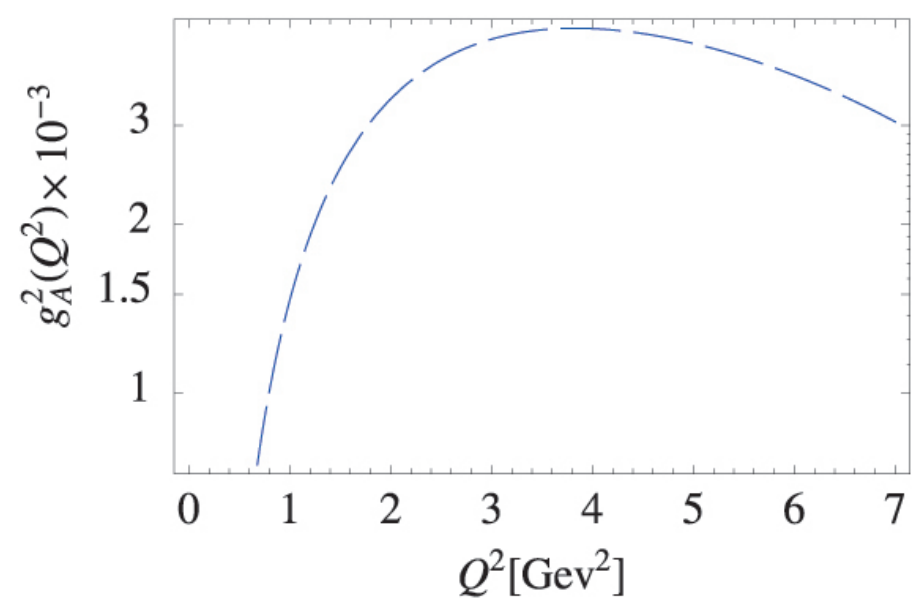

Figure 6: The anapole form factor $g_{A}^{2}\left(Q^{2}\right)$ from Eq. (4.1)as result of the $C P$-violation in the elastic $e p$ scattering in the region where the fit $R\left(Q^{2}\right)(4.5)$ is worked.

ton form factors. It is shown that the $C P$-violation effects give rise to the additional anapole proton form factor. The estimation of this form factor and the new values of the proton electric and magnetic form factors is performed in the region of the existing experiments. It is shown that the $C P$-violation effects lead to the small variation of the magnetic form factor and have strong influence on electric one.

\section{Acknowledgements}

Authors thank Professor V.E. Troitsky from SINP for helpfull discussions, the Program "Scientific and scientific-pedagogical specialist of innovation Russia" (Grant No 1338) and Ministry of Education and Science of Russian Federation (Grant No. 2.870.2011) for the support of our work and the Organizers of QFTHEP'2011 for kind invitation and hospitality.

\section{References}

[1] M. K. Jones et al., Phys. Rev. Lett. 84, 1398 (2002).

[2] O. Gayou et al., Phys. Rev. Lett. 88, 092301 (2002).

[3] O. Gayou et al., Phys. Rev. C. 64, 038202 (2001).

[4] M. E. Christy et al., Phys. Rev. C. 70, 015206, (2004).

[5] M. N. Rosenbluth, Phys. Rev. 79, 615 (1956).

[6] L. E. Price et al., Phys. Rev. D 4, 45 (1971).

[7] R. C. Walker et. al., Phys. Rev. D. 495671 (1994); L. Andivahis et. al., Phys. Rev. D. 50, 5491 (1994); A. F. Sill et. al., Phys. Rev. D. 48, 29 (1993); W. Bartel et. al., Nucl. Phys. B. 58, 429 (1973); L. E. Price et.al., Phys. Rev. D. 4, 45 (1971); J. Litt et. al., Phys. Lett. 31B, 40 (1970).

[8] C. E. Keppel et al., Jefferson Lab experiment No. E94-110, (1994).

[9] J. Arrington, Phys. Rev. C. 69, 032201 (2004) and arXiv:nucl-ex/0311019. 
[10] P. G. Blunden, W. Melnitchouk, and J. A. Tjon, Phys. Rev. C. 72, 034612 (2005).

[11] J. Arrington, W. Melnitchouk, and J. A. Tjon, Phys. Rev. C. 76, 035205 (2007).

[12] J. Bernstein, G. Feinberg and T. D. Lee, Phys. Rev. B1650, 139 (1965).

[13] V.M. Dubovik, A.A. Cheshkov, ZhETF. 51, 165 (1966).

[14] L. B. Okun, Sov. Phys. Usp. 9, 574 (1967).

[15] C. A. Baker et al., Phys. Rev. C. 72, 034612 (2005).

[16] Y. N. Srivastava et al., Phys. Rev. D. 82, 094003 (2010).

[17] A.F. Krutov, V.E. Troitsky, Phys. Rev. C. 68, 018501 (2003).

[18] A.A. Cheshkov, Yu. M. Shirokov, ZhETF. 44, 1983 (1963).

[19] A. F. Krutov, V. E. Troitsky, Phys. Part. Nucl. 40, 136 (2009).

[20] E. V. Balandina, N.P. Yudin, Vestnik MSU. 36, 14 (1995).

[21] E. L. Lomon, Phys. Rev. C. 64, 035204 (2001).

[22] M. Gari, W. Krumpelmann, Z. Phys. A. 322, 689 (1985). 